\title{
Aggregate formation and suspension culture of human pluripotent stem cells and differentiated progeny
}

Tracy A. Hookway ${ }^{\mathrm{a}}$, Jessica C. Butts ${ }^{\mathrm{a}, \mathrm{b}}$, Emily Lee ${ }^{\mathrm{c}}$, Hengli Tang ${ }^{\mathrm{c}}$, and Todd C. McDevitt ${ }^{\mathrm{a}, \mathrm{b}}$

${ }^{a}$ The Gladstone Institute of Cardiovascular Disease, San Francisco, CA

${ }^{b}$ Department of Bioengineering and Therapeutic Sciences, University of California San Francisco,

San Francisco, CA

${ }^{\mathrm{c} D}$ Department of Biological Science, Florida State University, Tallahassee, FL

Corresponding Author:

Todd C. McDevitt, Ph.D.

Senior Investigator, Gladstone Institute of Cardiovascular Disease

Professor, Dept. of Bioengineering \& Therapeutic Sciences, UCSF

1650 Owens Street

San Francisco, CA 94158

Email: todd.mcdevitt@gladstone.ucsf.edu

Phone: 415-734-2875 


\begin{abstract}
Culture of human pluripotent stem cells (hPSC) as in vitro multicellular aggregates has been increasingly used as a method to model early embryonic development. Three-dimensional assemblies of hPSCs facilitate interactions between cells and their microenvironment to promote morphogenesis, analogous to the multicellular organization that accompanies embryogenesis. In this paper, we describe a method for reproducibly generating and maintaining populations of homogeneous three-dimensional hPSC aggregates using forced aggregation and rotary orbital suspension culture. We propose solutions to several challenges associated with the consistent formation and extended culture of cell spheroids generated from hPSCs and their differentiated progeny. Further, we provide examples to demonstrate how aggregation can be used as a tool to select specific subpopulations of cells to create homotypic spheroids, or as a means to introduce multiple cell types to create heterotypic tissue constructs. Finally, we demonstrate that the aggregation and rotary suspension method can be used to support culture and maintenance of hPSC-derived cell populations representing each of the three germ layers, underscoring the utility of this platform for culturing many different cell types.
\end{abstract}

\title{
Keywords (max 6)
}

- Cell aggregation

- 3D tissue culture

- Suspension culture

- Human pluripotent cells 


\subsection{Introduction}

Cell culture technologies developed in recent years recapitulate in vivo cellular microenvironments with increasing sophistication and complexity by incorporating a variety of signals found within the environment of native tissues. Such in vitro models span a range from 2D monolayer approaches to more complex 3D tissue engineering strategies. Traditional 2D culture methods offer a straight-forward approach to enable studies which parse out the relative influence of individual stimuli on cell populations while not being limited by metabolic or oxygen transport constraints. However, adherent monolayer methods do not fully account for the complex interactions that occur between cells and their three-dimensional extracellular environment. While large engineered tissues and organs require many cells, three-dimensional microscale constructs can faithfully generate analogous multicellular tissue architecture and allow for the interrogation of 3D interactions of multiple cell populations, as well as the assessment of physiological functional parameters, with minimal numbers of cells.

In vitro culture of cell aggregates (micron-scale tissue constructs) recreates the biochemical and biophysical microenvironment of native tissues defined by the intercellular adhesions [1], extracellular matrix, and signaling between neighboring cells [2-4]. Human pluripotent stem cells (hPSCs) are often cultured as 3D spheroids to initiate "organoid" formation that mimics many aspects of embryonic development including morphological and phenotypic transitions, such as primitive streak formation and embryonic gastrulation [5], including the epithelial-tomesenchymal transition and accompanying alterations in the extracellular matrix $[6,7]$. Traditionally, aggregates formed from PSCs are used to promote coincident differentiation into multiple germ layers and can be used as a surrogate in vitro test of pluripotency analogous to in vivo teratoma assays [8]. Generating multicellular aggregates from single cell suspensions of mouse pluripotent cells is relatively straight-forward, however, similar aggregation of human pluripotent cells has historically been more challenging due to the decreased viability that arises 
following dissociation of hPSC colonies into single cells [9]. However, the addition of Rhoassociated protein kinase (ROCK) inhibitors, which enhance survival of single cells by preventing dissociation-induced apoptosis, has enabled aggregate formation from single cell suspensions of hESCs and hiPSCs [10,11], suggesting similar intrinsic cell adhesion mechanisms between the two cell types. Despite reported differences in their epigenetic profiles [12], direct comparative studies between human ESCs and iPSCs have demonstrated similarities in gene expression [13], morphological appearance [14], and differentiation potential [15], raising the question as to whether both cell types aggregate and differentiate similarly as 3D multicellular aggregates.

More recently, the culture of 3D spheroids has been adopted and expanded for a variety of cell types differentiated from hPSCs such as "neurospheres" derived from neural stem cells and “cardiospheres" derived from stem cell-derived cardiomyocytes [16, 17]. Microtissues generated by the aggregation and self-assembly of numerous cell types results in homotypic and/or heterotypic spheroids that can more faithfully recapitulate phenotypic and functional traits of analogous native tissues. One recent example of functional heterotypic hPSC-derived selfassembly is the formation of vascularized iPS-derived liver buds. A combination of iPSC-derived hepatocytes, mesenchymal stem cells, and endothelial cells aggregated together to form a functional unit that integrated with host vasculature upon implantation in vivo and produced albumin for an extended period of time [18, 19]. This example highlights the phenotypic and functional similarities to native tissue that can be achieved by self-assembly of cells into microscale tissues.

This paper describes a method to rapidly generate and culture homogeneous hPSC spheroid populations using microscale aggregation technologies combined with simple hydrodynamic culture environments. We discuss some of the common challenges that accompany specific steps 
of this process and suggestions for adapting this robust culture platform to other cell types. In addition, we demonstrate how this $3 \mathrm{D}$ aggregate culture method may be used for selection and enrichment of specific cell types and can be used to controllably form homotypic or heterotypic constructs composed of multiple cell types. Finally, we demonstrate the robust nature of these methods to yield spheroids composed of hPSC-derived cells from each of the 3 germ layers and expressing the appropriate phenotypic markers, demonstrating the utility of this platform across a variety of relevant cell types of interest.

\subsection{Materials and Methods}

\subsection{Cell culture}

\subsubsection{Pluripotent stem cells}

Human embryonic stem cells (H7 and H9; WiCell, Madison, WI) and human induced pluripotent cells (WTC11 and 903-20, generously provided by Bruce Conklin and Chunhui Xu, respectively) were maintained in an undifferentiated state on Matrigel ( $80 \mu \mathrm{g} / \mathrm{ml}$, Corning Inc., Corning, NY) coated polystyrene tissue culture treated dishes in mTeSR or Essential 8 media (Stem Cell Technologies, Vancouver, BC). Both ESCs and iPSCs were routinely passaged either as single cells or as colonies prior to reaching $80 \%$ confluence. To passage as single cells, hPSCs were treated with Accutase (Innovative Cell Technologies, San Diego, CA) for 5 minutes at $37^{\circ} \mathrm{C}$, diluted with an equal volume of PBS, collected in a conical tube, centrifuged at $200 \mathrm{rcf}$ for 5

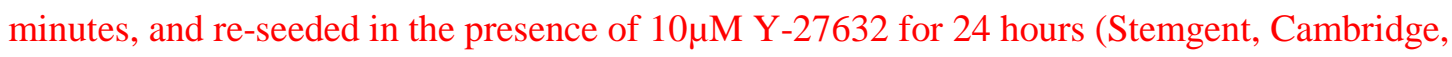
MA) to promote cell survival. To passage hPSCs as colonies, plates were treated with Dispase (Stem Cell Technologies) for 7 minutes at $37^{\circ} \mathrm{C}$, washed three times with DMEM/F12 to remove residual Dispase, manually scraped with a cell lifter to detach from the bottom of the plates as

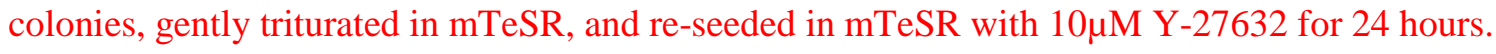

\subsubsection{Cardiac fibroblasts}


Cardiac fibroblasts (Cell Applications, San Diego, CA) were cultured on tissue culture

polystyrene in Cardiac Fibroblast Growth Media (Cell Applications). Fibroblasts were passaged at $80 \%$ confluence using $0.25 \%$ Trypsin $/ 2.21 \mathrm{mM}$ EDTA (Corning) at $37^{\circ} \mathrm{C}$ for 5 minutes, quenched with an equal volume of DMEM (Corning) containing 10\% Fetal Bovine Serum (Hyclone), centrifuged at $200 \mathrm{rcf}$ for 5 minutes, and seeded at 7,000 cells $/ \mathrm{cm}^{2}$. Fibroblasts from passages 4-7 were used for heterotypic seeding experiments.

\subsection{Aggregate formation and rotary suspension culture}

We fabricated two different master templates corresponding to $400 \mathrm{x} 400 \mu \mathrm{m}$ and $800 \mathrm{x} 800 \mu \mathrm{m}$ sized microwells by conventional photolithographic processes. Briefly, photoresist was deposited on silicon wafers and selectively removed from the regions where microwells were to be formed. The wafers were then chemically etched with $45 \%$ potassium hydroxide (w/w, BDH Chemicals) for 18-24 hours to generate inverted pyramidal arrays with side walls of either $400 \mu \mathrm{m}$ or 800 $\mu \mathrm{m}$, similar to previously published protocols [20]. The silicon wafers were used as templates for replica molding of polydimethylsiloxane (PDMS; Sylgard-184, Dow Corning) or 2\% (w/v) agarose (OmniPur, EMD Millipore, Billerica, MA). PDMS was mixed at a 10:1 ratio (base:curing agent), degassed for 30 minutes, poured onto the silanized silicon wafer, degassed again until all bubbles were removed from the microwells, and cured at $60^{\circ} \mathrm{C}$ for 12 hours. PDMS microwell inserts were fabricated by punching out individual $15 \mathrm{~mm}$ circles that were autoclaved and placed in the wells of a 24-well plate. For agarose inserts, an inverse PDMS template was generated containing $15 \mathrm{~mm}$ circular wells with inverse microwell geometries. Autoclaved agarose was pipetted into each $15 \mathrm{~mm}$ circular well of the sterilized inverse PDMS template, generating agarose microwells. Upon gelation of the agarose, the microwells were transferred to 24-well plates. Media was added to each well $(500 \mu \mathrm{l})$ and 24 -well plates were centrifuged at $2000 \mathrm{rcf}$ to remove any air bubbles. Cell aggregates were generated by dissociating PSC cultures into single cell suspensions with Accutase for 3-5 minutes followed by centrifugation (200 rcf) 
into microwells at a density that ranged from 300-10,000 cells in 1-2 $\mathrm{mL}$ culture medium per microwell. After 18-24 hours of formation, 3D aggregates were transferred to $100 \mathrm{~mm}$ non-tissue culture treated dishes (100-5,000 aggregates/plate) along with $10 \mathrm{~mL}$ of culture media and maintained for up to 21 days on a rotary orbital shaker (Benchmark, $65 \mathrm{rpm}$ ) as described previously [21]. (*Note*: Both the number of cells/aggregate and the number of aggregates/mL media can be modulated using this system to achieve the desired cell density. However, we have empirically observed the most consistent results with a minimum starting cell density of at least $2 \times 10^{5}$ cells/mL to maintain the health and growth of the cell populations) [21].

\subsection{Directed differentiations}

\subsubsection{Cardiomyocytes}

Differentiation of cardiomyocytes from hPSCs was achieved with a serum-free, chemically defined protocol on monolayer cultures using a serum-free medium [22]. Briefly, hPSCs (H7, WiCell; 903-20 hiPSCs, generously donated by Dr. Chunhui Xu) were seeded onto Matrigel coated dishes in mTeSR medium (Stem Cell Technnologies) and grown to confluence for 3 days. On day 0 of differentiation, the cultures were treated with RPMI 1640 medium (Corning) supplemented with B27 Supplement minus insulin (Life Technologies, Grand Island, NY) and 12 $\mu$ M CHIR99021 (Selleckchem, Houston, TX). The initial induction media was completely removed after 24 hours and exchanged with RPMI/B27 minus insulin. On day 3 of differentiation, the media was changed again to RPMI/B27 minus insulin (Life Technologies) supplemented with $5 \mu \mathrm{M}$ IWP2 (Tocris, Bristol, UK) for 48 hours. Cells were then maintained with media changes every 3 days for 14 days in RPMI/B-27 with insulin (Life Technologies).

\subsubsection{Hepatocytes}

Human ESCs were differentiated into hepatic cells in 2D monolayer culture as previously described [23]. Briefly, ESCs were seeded onto Geltrex (Invitrogen, Carlsbad, CA) coated dishes 
in Stem Pro medium (Invitrogen) at 30-40\% confluence. On day 1 of differentiation, medium was changed to Defined Media (DM; DMEM/F12 containing 10\% Probumin (EMD Millipore), 0.2\% $\beta$-Mercaptoethanol (Sigma), 1\% L-Alanyl-L-Glutamine (Invitrogen), 2\% hESC supplement augmented with 100 ng/ml Activin-A (R\&D Systems, Minneapolis, MN), 8 ng/ml b-FGF (Invitrogen), and $25 \mathrm{ng} / \mathrm{ml} \mathrm{Wnt-3A} \mathrm{(Stemgent).} \mathrm{On} \mathrm{days} \mathrm{2-4} \mathrm{of} \mathrm{differentiation,} \mathrm{cells} \mathrm{were}$ exposed to DM supplemented with $100 \mathrm{ng} / \mathrm{ml}$ Activin-A and $8 \mathrm{ng} / \mathrm{ml}$ b-FGF. On days 5-7 media was changed to DM supplemented with only $50 \mathrm{ng} / \mathrm{ml}$ b-FGF. On day 8 of the differentiation media was changed to DM supplemented with 50 ng/ml FGF-10 (PeproTech, Rocky Hill, NJ), $0.1 \mu \mathrm{M}$ retinoic acid (Sigma), $1 \mu \mathrm{M}$ SB431542 (Sigma). On day 9, differentiated human hepatocyte $(\mathrm{DHH})$ monolayers were dissociated by Accutase and seeded into agarose microwell

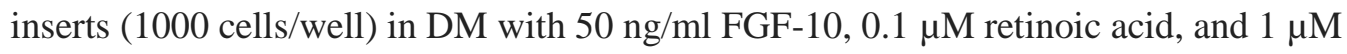
SB431542 for 20 hours. Following aggregation, spheroids were re-suspended in DM supplemented with 30 ng/ml FGF-4, 50 ng/ml EGF (PeproTech), and 50 ng/ml HGF (PeproTech) and either transferred to $100 \mathrm{~mm}$ low adherent culture dishes (2,000 aggregates in $10 \mathrm{~mL}$ culture media) for rotary culture or reseeded into fresh agarose-inserts (500 aggregates in $2.5 \mathrm{~mL}$ culture media) for static culture. Media was changed every two days for one week.

\subsubsection{Motor Neurons}

Human motor neuron differentiation was achieved as previously described [17]. Briefly, aggregates were formed via forced aggregation of human PSCs (10,000 cells per aggregate) into $800 \mu \mathrm{m}$ microwells. Aggregates were cultured in mTeSR media with dual SMAD inhibition (10 $\mu \mathrm{M}$ SB 435142 and $0.2 \mu \mathrm{M}$ LDN 193189, Stemgent, Cambridge, Massachusetts) for 5 days with media changes every 2 days. The medium was then changed to DMEM F12 supplemented with N2 (Life Technologies) and $1 \mu \mathrm{M}$ retinoic acid (Sigma Aldrich), $0.4 \mu \mathrm{g} / \mathrm{ml}$ ascorbic acid (Sigma Aldrich), and $10 \mathrm{ng} / \mathrm{ml}$ brain derived neurotrophic factor (R\&D, Minneapolis, Minnesota) was added. On day 7, $1 \mu \mathrm{M}$ purmorphamine, a sonic hedgehog agonist (Millipore, Hayward, 
California) was added to the other signaling molecules. Media was changed every 2 to 3 days and cultured for 17 days total to obtain a motor neuron phenotype.

\subsection{Histology and immunohistochemical staining}

Cell aggregates were fixed for 1 hour with $10 \%$ neutral buffered formalin for histology, or $4 \%$ paraformaldehyde for whole mount staining. Histological samples were embedded in Histogel (Thermo Scientific) and dehydrated through a series of graded ethanols, xylene, and paraffin before embedding into paraffin blocks. Five micron sections were cut and adhered to charged glass slides. Sections were then stained with hematoxylin and eosin or alcian blue with nuclear fast red counterstain (Newcomer Supply, Middletown, WI). For immunohistochemical staining, sections were deparaffinized, rehydrated, blocked in $1.5 \%$ donkey serum, and incubated with primary antibodies (Oct4, Santa Cruz Biotechnology, 1:100; and Albumin, Sigma Aldrich, 1:100) overnight at $4{ }^{\circ} \mathrm{C}$. AlexaFluor 488 conjugated donkey anti-goat IgG antibody (1:400, Life Technologies) was used at room temperature for 1 hour to detect Oct4 prior to counterstaining with Hoechst (Sigma Aldrich). Albumin was detected using the ImmPRESS HRP Polymer Detection Kit (Vector Laboratories, Burlingame, CA) and counterstained with hematoxylin. Stained sections were imaged on a Nikon 80i upright microscope outfitted with a SpotFLEX camera (Diagnostic Instruments Inc.). For whole mount staining, fixed aggregates were permeabilized with (1.5\% triton $\mathrm{x}-100$ for 1 hour, blocked with $1.5 \%$ normal donkey serum for 4 hours, and incubated with primary antibodies overnight at $4{ }^{\circ} \mathrm{C}(\alpha$-actinin, Sigma, 1:1000; $\beta$-IIItubulin, BioLegend, 1:1000). Aggregates were then washed three times with PBS and incubated with secondary antibodies (AlexaFluor 546 conjugated donkey anti mouse IgG and donkey anti rabbit IgG, 1:400, Life Technologies).

\subsection{Morphometric analysis of cell aggregates}


Phase images of cardiospheres were acquired after 7 days of culture with an EVOS FL Cell imaging system (EVOS). Average cell aggregate diameter was measured using ImageJ analysis software. A minimum of 150 aggregates from each sample were measured. Measurements are represented as boxplots to show the spread of data where the boxes indicate the first, second, and third quartiles and whiskers indicate the maximum and minimum values. The normality of the distribution was examined by a D'Agostino and Pearson test and statistical significance between groups was calculated using a Wilcoxon Rank Test , p<0.05 (Prism, GraphPad Software, Inc.).

\subsection{Results and Discussion}

\subsection{Human embryonic stem cells and induced pluripotent stem cells aggregate similarly}

To investigate the ability of various hPSC sources to readily aggregate, we cultured multiple lines of human ESCs (H7s and H9s) and iPSCs (WTC11 and 903-20) for several passages prior to aggregation in either mTeSR or E8 medium using either single cell passaging or colony passaging methods. Each of the cell lines examined grew at comparable rates and exhibited similar compact colony morphologies (Fig.1 A, B). To form aggregates, all cell lines were dissociated with

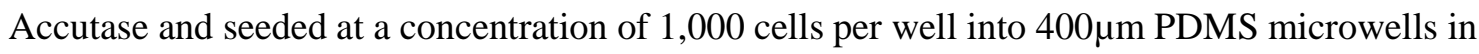
the presence of ROCK inhibitor (10 $\mu \mathrm{M} \mathrm{Y-27632).} \mathrm{Each} \mathrm{of} \mathrm{the} \mathrm{hPSC} \mathrm{lines} \mathrm{aggregated} \mathrm{within} 18$ hours to generate uniform, round spheroids (Fig.1 D, E), indicating that independent hPSC lines did not appear to have any noticeable effects on the kinetics of aggregation. In all cell lines, the majority of cells incorporated into the spheroids, however, there were often a number of cells that settled within the wells but were not incorporated within the resulting aggregates; unincorporated cells generally resided right beneath the aggregates with a rounded morphology (black arrowheads, Figure 1C and F). A representative image of typical hPSC aggregation is shown in Figure $1 \mathrm{C}$ where clearly distinct spherical edges are observed around the aggregates within the microwells (white arrowhead, Figure 1C). However, on some occasions, variable spheroid formation efficiencies could be observed between seeding experiments under seemingly parallel 
conditions, demonstrated by the inefficient aggregation of hESCs (H7) in Figure 1F.

Interestingly, hPSCs that were pre-cultured using both single cell passaging and colony passaging appeared to similarly aggregate into spheroids, suggesting that pre-adaptation of cells to dissociation is not required for cells to aggregate into spheroids[24].

Based on numerous seeding experiments we have identified several key criteria to help optimize consistent hPSC aggregation within microwells. Specific requirements may vary between cell lines, but a minimum concentration of $10 \mu \mathrm{M}$ ROCK inhibitor provided the most consistent hPSC aggregation results. In fact, some cell lines (such as $\mathrm{H} 7$ ) benefit from a pre-treatment of 5-10 $\mu \mathrm{M}$ rock inhibitor of the $2 \mathrm{D}$ cultures prior to dissociation to achieve the best aggregation results. Additionally, microwell seeding density and initial aggregate size can affect the consistency of formation. We have seeded a wide range of hPSCs (300-10,000 cells per aggregate), however, the most consistent aggregation into uniform cell spheroids was observed with a minimum cell seeding density of 1,000 cells per aggregate. Together our results suggest that uniform populations of homogeneous aggregates can be readily formed from seemingly any hPSC line using microwells that promote high cell density aggregation, although the optimal formation requirements for each specific line may differ slightly.

\subsection{Rotary orbital suspension culture promotes maintenance of homogeneous populations of} hPSC aggregates

Following the initial aggregation of hPSCs to form spheroids, the next consideration is the maintenance platform required for culturing the cell aggregates for prolonged periods of time in 3D. To date there are a variety of methods described in the literature for culturing aggregates, ranging from simply maintaining spheroids in low-adherent tissue culture plates under static conditions [25, 26], to culturing in larger-volume hydrodynamic bioreactors [27-30]. While ultimate scale-up needs for some clinical applications may require large bioreactors to achieve 
upward of $10^{9}$ cells/patient [31, 32], scientific experiments do not typically require the same magnitude of scale-up. To this end, we have developed a rotary orbital suspension protocol that can be used to culture cell aggregates on the scale of $10^{5}-10^{6}$ cells $/ \mathrm{mL}$, which is sufficient for the most common forms of experimental analyses (e.g. gene expression, flow cytometry, biochemical assays, etc.).

To transfer cell spheroids to suspension culture, aggregates were gently pipetted with wide orifice $1 \mathrm{~mL}$ pipet tips to dislodge them from the microwells and transferred to $100 \mathrm{~mm}$ non-adherent culture dishes in a total of $10 \mathrm{~mL}$ of media. Dishes were then stacked vertically and placed on a rotary orbital shaker in a cell culture incubator (Figure 2A and Supplementary Video 1). Cell aggregates were initially dispersed throughout the dish as shown in Figure 2B (black arrowheads), but quickly gathered to the center of the dishes within 5 seconds when exposed to rotary conditions at either 40 or $65 \mathrm{rpm}$ (Supplementary Video 2). Altering rotary speed impacted the hydrodynamic environment causing the aggregates to gather in the center of the dishes in different densities (white arrowheads, Figure 2C and D), where higher rotary speed forced aggregates to congregate more tightly in the center of the dish. Relatively homogeneous populations of human PSC aggregates were maintained in suspension with the hydrodynamic forces exerted by rotary orbital culture. However, whereas $45 \mathrm{rpm}$ is sufficient to maintain mouse EBs in culture without agglomeration [21, 33, 34], a higher rotary speed of $65 \mathrm{rpm}$ was typically necessary to prevent agglomeration of human PSC aggregates at a similar aggregate density (200 aggregates $/ \mathrm{mL})$.

Another critical determinant in the successful maintenance and culture of hPSC spheroids is the aggregate density. As described above, cell aggregates can be generated from a range of 30010,000 cells per aggregate. Individual culture dishes (e.g. $100 \mathrm{~mm}$ diameter) on the rotary orbital shakers can accommodate a large range of aggregate numbers (we have tried 100's-1,000's) 
leading to a potential wide range of total cells within the system. We have had more success and achieved more consistent results by culturing hPSC aggregates at higher densities $(>1,000$ aggregates per dish), where there appears to be a critical threshold below which the cell aggregates do not increase in size, dissociate overtime, and ultimately yield significantly fewer viable cells. However, there also appears to be a maximum density above which the culture media gets depleted too quickly, resulting in the need for frequent feeding to avoid negative effects on cell viability.

While optimization of specific conditions for different cell lines may be required for the best results, using the rotary suspension platform during the culture of $3 \mathrm{D}$ aggregates enables the growth and maintenance of uniform populations of EBs in large quantities. This straight-forward hydrodynamic culturing system is easily integrated into standard cell culture incubators and practices performed by biomedical research laboratories to enable studies of hPSC differentiation, embryonic development, and ultimately more complex microtissue formation.

\subsection{Human embryoid bodies spontaneously differentiate under serum-free conditions}

Using the combined technique of forced aggregation and rotary suspension culture, our lab has examined many aspects of mouse ESC differentiation in the context of 3D embryoid bodies [21, $33,34]$. In doing so, we demonstrated that it is possible to achieve more reproducible differentiation results for homogeneously sized EB populations both within and between experiments performed under similar conditions. Thus, in order to directly examine how this culture platform impacts the formation and maintenance of human EBs, we monitored spontaneous differentiation of hESC-derived EBs over the course of 2 weeks in suspension culture. 
Adherent colonies of undifferentiated hPSCs (H7 cells) were dissociated, aggregated into $400 \mu \mathrm{m}$ PDMS microwells (1,000 cells per well), and then cultured for 14 days in rotary orbital suspension culture $(65 \mathrm{rpm})$ in serum-free medium. As expected, the resulting EBs increased in size over the course of 2 weeks, from a diameter at day 1 of culture of $\sim 150 \mu \mathrm{m}$ to $\sim 300 \mu \mathrm{m}$ after 4 days. The observed increase in size over the first 4 days in culture is due to a combination of cell proliferation and morphological changes, such as cystic formation, occurring within the tissues that affects the packing density of the cells. As demonstrated in Figure 3, the aggregates adopted a more differentiated morphology by day 4 of culture, indicated by the cystic cavities that developed on the interior of the EBs as well as tightly packed epithelial-like regions (Fig. 3 A, E, I, M). Consistent with the GAG-rich extracellular matrix of undifferentiated ESCs [35], the EBs exhibited positive alcian blue staining throughout differentiation, and particularly in the interstitial space in regions with lower cell densities and more mesenchymal appearance (Fig. 3 $\mathrm{B}, \mathrm{F}, \mathrm{J}, \mathrm{N})$. The undifferentiated ESC marker, Oct4, was expressed by cells in regions of densely packed nuclei throughout the EBs at early time points (Days 4-7), but decreased in expression over time, with few Oct4+ cells visible by Days 10-14 of differentiation (Fig. 3 C, G, K, O), further indicating loss of pluripotency and progressive differentiation. Finally, 3D morphogenesis is often accompanied by dynamic rearrangement of cellular adhesions and cytoskeletal proteins[36], reflected in differentiating stem cell aggregates by changes in the spatial distribution of actin over time (Fig. 3 D, H, L, P). On day 4 of differentiation, the aggregates were densely packed and exhibited only cortical actin structures, whereas by day 7 of differentiation, prominent actin fibers were evident throughout the cells within the aggregates (Fig. 3 D, H, L, P insets), demonstrating there were fundamental cytoskeletal structural changes that occur within the 3D aggregates over time. 
In addition to forming aggregates directly from hPSCs, we also generated microtissues from predifferentiated hPSC-derived cardiomyocytes using forced aggregation and rotary suspension culture. To achieve this, we followed a previously published and well established 2D monolayer directed differentiation protocol that temporally modulates Wnt signaling in order to achieve spontaneously beating cardiomyocyte populations within 14 days [22, 37]. Despite this robust directed differentiation protocol, variability in cardiomyocyte efficiency was observed between experiments ranging from 10\%-80\% cardiomyocytes. Regardless of the differentiation efficiency, the resulting heterogeneous cardiomyogenic mixture of adherent cells were dissociated and aggregated into microwells for 24 hours. We observed a correlative relationship between the resulting size of the cardiospheres and the initial cardiomyocyte efficiency. Larger aggregates were formed from differentiated cultures that yielded a greater initial percentage of cardiomyocytes (Figure 4), compared to smaller aggregates generated from less efficient differentiations.

We have recently demonstrated that hPSC-derived cardiomyocyte (hPSC-CM) spheroids enrich cardiomyocyte populations to $>90 \%$, excluding other cell types that arose during the previous 2week differentiation period [16]. The enrichment of cardiomyocytes occurred regardless of the initial differentiation purity (generally between 10-40\%), however, an association between the resulting size of the cardiosphere and the initial purity of the differentiation was observed. Aggregation of specific cell types is directly related to the cells' adhesion protein profile mediated by the collective ensemble of cell surface receptors [38], suggesting cell-mediated aggregation may be a simple and efficient method for self-selecting certain sub-populations from heterotypic cultures. These results suggest that $3 \mathrm{D}$ multicellular aggregation can be used to specifically isolate certain cell types from heterogeneous differentiated cultures based on principles analogous to the differential adhesion hypothesis $[39,40]$. 


\subsection{Forced aggregation enables heterotypic microtissue generation}

Although aggregation can be used as an experimental tool to isolate individual cell populations as described above, most functional tissues are not comprised of a single homogeneous cell population naturally in vivo. Rather, each tissue is comprised of multiple cell types working together in concert to enable physiological function. Therefore, the ability to generate microtissues with multiple cell types is paramount to generating more accurate tissue models for in vitro analytical assays, as well as potentially regenerative medicine applications [41, 42]. To determine if multiple cell types could co-aggregate together to intentionally form a heterotypic yet controlled spheroid, hPSC-CMs were combined with a supporting stromal cell population, cardiac fibroblasts $(\mathrm{CF})$, within the microwells. Prior to seeding, hPSC-CMs were pre-labeled with CellTracker Green ${ }^{\mathrm{TM}}$ and cardiac fibroblasts were pre-labeled with CellTracker Red ${ }^{\mathrm{TM}}$. The two cell types were combined at varying ratios (hPSC-CM:CF, 100:0, 75:25, 50:50, 25:75, and 0:100), seeded into the microwells to aggregate for 24 hours, and cultured in suspension for 3 days thereafter. Co-seeded cells at all ratios aggregated to form microtissues, and fluorescent microscopy reflected a decrease in the frequency of CellTracker Green ${ }^{\mathrm{TM}}+$ cells as the ratio of hPSC-CM:fibroblasts decreased, as would be expected (Fig 5). The resulting aggregates that were comprised of mostly or all hPSC-CMs (75:25 or 100:0) appeared slightly smaller than the aggregates containing greater numbers of fibroblasts. This is potentially due to the fact that the hPSC-CM differentiated cells were not a pure population and were not sorted prior to aggregation. As described in the previous section, aggregation of heterogeneous hPSC-CM cultures largely excludes the non-myocyte cell fractions, thus the same heterogeneous aggregation was applied in this study, which led to smaller aggregates in groups with higher hPSC-CM ratios. Alternatively, the fact that cardiac fibroblasts proliferate more than hPSC-CM may account for small size differences between the groups after 3 days of culture, leading to overall larger aggregates with higher percentages of fibroblasts. 
Although these mixing studies were simply intended to demonstrate the technical feasibility of forcibly combining different cell types, the incorporation of stromal cells within cardiomyocyte aggregates may have long-term phenotypic consequences that we and others are interested in characterizing. In vivo, cardiac fibroblasts produce extracellular matrix to support the myocardium, secrete paracrine factors to influence cardiomyocyte behavior, and electrically couple with cardiomyocytes to allow for continuous electrical signal throughout the heart. Inclusion of supporting cell populations within engineered cardiac tissues has been shown to improve tissue strength, promote cardiomyocyte survival, and induce early signs of cardiomyocyte maturation [41]. Many approaches have been used to mix cell populations in engineered tissues, but this study demonstrates that cellular assembly of heterotypic cultures can be achieved by simply mixing two cell types at the onset of aggregation. Intentional introduction of mature cardiac fibroblasts and incorporation within the aggregates suggests that the hPSC-CMs and the fibroblasts share similar cell adhesion markers allowing them to readily co-aggregate together [43]. Specific pairings of cells that typically exist together (such as cardiomyocytes and cardiac fibroblasts) may be more capable of mixing more easily than distinct cell types that arise independently during in vitro differentiation in parallel.

\subsection{Various human PSC-derived cells self-assemble to form microtissues}

Cell aggregation is a useful platform for generating multicellular constructs from human PSCs, but in order to become a widely adopted method it must support the differentiation, culture, and maintenance of many differentiated cell types. Therefore, we investigated the parameters necessary to aggregate various hPSC-derived cell populations and maintain differentiated cell aggregates representing tissues from each germ layer (endoderm, mesoderm, and ectoderm). Initially, we used previously established protocols to differentiate the cells into hepatocytes and cardiomyocytes in 2D monolayer cultures and then formed 3D aggregates. In contrast, motor neurons were differentiated as 3D aggregates in suspension culture, similar to previous reports 
$[17,22,23,37]$. Interestingly, each of these cell types required different parameters for the successful generation and culture of 3D spheroids (Fig 6). Similar to what has been described in previous studies, aggregate size played a critical role in supporting the differentiation, viability, and maintenance aggregates over the course of culture [44-46]. The primary difference between cell types was the number of cells required to consistently generate uniform sized aggregates that could remain viable in culture for at least one week (Fig 6 A,B,C). Hepatic and cardiac aggregates formed and were maintained well when seeded into $400 \mu \mathrm{m}$ wide microwells at a density 1,000-2,000 cells per microwell, resulting in aggregates that expressed specific functional (albumin) and phenotypic (actinin) markers of their respective cell types (Fig 6 A,B, D, E).

However, neural differentiations were more consistent using larger microwells ( $800 \mu \mathrm{m}$ wide) that accommodate 10,000 cell aggregates to enable efficient and consistent generation of $\beta$-III-tubulin positive cells within the constructs (Fig $6 \mathrm{C}, \mathrm{F}, \mathrm{I})$. This difference in aggregate size may be related to the relative adhesivity of different cell types for each other or the ability and kinetics of extracellular matrix production to help stabilize the aggregates after initial formation. In addition, paracrine signaling between cells may play a role in determining the necessary size of aggregates for successful tissue culture given that some cell types may require signals provided by adjacent cells. The size of the aggregates may be related to the survival of cells throughout various differentiation protocols. In the examples outlined here, both the cardiomyocytes and the differentiated hepatocyte-like cells were pre-differentiated in 2D prior to aggregate formation. Although cell death is observed during 2D culture after treatment with small molecules, the 3D aggregation is performed afterward, when cell loss is minimal during initial aggregate formation. However, the motor neuron differentiation described was initiated after initial aggregate formation and significant cell death was observed starting on day 7 of differentiation after the addition of purmorphamine. Thus, a higher initial starting density was required to prevent the aggregates from disintegrating due to excessive cell death. 
Another consideration when generating aggregates from differentiated cell types is the material from which the microwells are made. As described in the methods, both PDMS and agarose were used to create microwell arrays. The PDMS wells were similar to the commercially available Aggrewell inserts (Stemcell Technologies) and support the aggregation of several cell types, including cardiac and neural cells. Differentiated human hepatocyte-like aggregates, however, are difficult to remove from the PDMS microwells and transfer to rotary culture because individual cells adhere not only to each other, but also attach to the surface of the PDMS, thus anchoring the aggregates within the wells. In an effort to decrease the cell adhesion to the material surface, replica molding was used to generate agarose microwells. The agarose microwells yield comparable aggregation to the PDMS, but are a less-adhesive material, thereby allowing more adhesive cell populations to aggregate readily without attaching to the wells. The ability of various cell types to consistently aggregate in the microwells appears to be a balance between cell-to-cell adhesion forces and cell-to-microwell substrate adhesion forces. As hPSCs differentiate their adhesion properties change dramatically [47], making it necessary to utilize different materials with low cell adhesion to generate the microwells.

Finally, variability is inherent in any biological system, however, this cellular aggregation platform is robust and allows for tissue formation under a broad range of parameters. Even though differentiation efficiencies can vary widely between labs, users, or even experiments, multicellular aggregation of PSCs and differentiated cell types can be reliably achieved, regardless of differentiation efficiency [16].

\subsection{Conclusions}

In this paper we demonstrate that forced aggregation and rotary suspension culture can be used to support differentiation and maintenance of hPSC cells and many differentiated progeny. We identify several challenges associated with the consistent generation of hPSC derived spheroids and parameters to consider (including microwell size, microwell material, number of cells per 
aggregate, aggregate density, rotary orbital speed, etc.) when adapting this strategy to new cell lines or differentiated cell types. Using this 3D culture platform, we demonstrate how to selectively form homotypic or heterotypic aggregates based on either selection of specific cell populations or intentional introduction of multiple cell types. Finally, we use this multicellular aggregate based approach to form and maintain robust populations of spheroids composed of hPSC-derived cells representing each germ layer. Taken together, these data indicate that multicellular spheroids can be generated from most hPSC lines and differentiated progeny, and thus can be used as a robust model to study early embryonic mammalian development.

\subsection{Acknowledgements}

This work was supported by funding from the California Institute for Regenerative Medicine (LA1-08015) and the National Science Foundation (NSF) (CBET 0939511). T.A.H. is currently supported by an American Heart Association (AHA) Postdoctoral Fellowship. The authors would like to thank Drs. Bruce Conklin and Chunhui Xu for generously donating human induced pluripotent stem cells (WTC11 and 903-20 lines) that were used for many of these studies.

\subsection{References}

1. van den Brink, S.C., et al., Symmetry breaking, germ layer specification and axial organisation in aggregates of mouse embryonic stem cells. Development, 2014. 141(22): p. 4231-42.

2. Evseenko, D., et al., Identification of the critical extracellular matrix proteins that promote human embryonic stem cell assembly. Stem Cells Dev, 2009. 18(6): p. 919-28.

3. Akins, R.E., Jr., et al., Three-dimensional culture alters primary cardiac cell phenotype. Tissue Eng Part A, 2010. 16(2): p. 629-41.

4. Chang, T.T. and M. Hughes-Fulford, Monolayer and spheroid culture of human liver hepatocellular carcinoma cell line cells demonstrate distinct global gene expression patterns and functional phenotypes. Tissue Eng Part A, 2009. 15(3): p. 559-67.

5. $\quad$ Fuchs, C., et al., Self-organization phenomena in embryonic stem cell-derived embryoid bodies: axis formation and breaking of symmetry during cardiomyogenesis. Cells Tissues Organs, 2012. 195(5): p. 377-91.

6. Chan, D.N., et al., PTK7 marks the first human developmental EMT in vitro. PLoS One, 2012. 7(11): p. e50432. 
7. Rust, W.L., A. Sadasivam, and N.R. Dunn, Three-dimensional extracellular matrix stimulates gastrulation-like events in human embryoid bodies. Stem Cells Dev, 2006. 15(6): p. 889-904.

8. Sheridan, S.D., V. Surampudi, and R.R. Rao, Analysis of embryoid bodies derived from human induced pluripotent stem cells as a means to assess pluripotency. Stem Cells Int, 2012. 2012: p. 738910.

9. Sjogren-Jansson, E., et al., Large-scale propagation of four undifferentiated human embryonic stem cell lines in a feeder-free culture system. Dev Dyn, 2005. 233(4): p. 1304-14.

10. $\mathrm{Xu}, \mathrm{Y}$., et al., Revealing a core signaling regulatory mechanism for pluripotent stem cell survival and self-renewal by small molecules. Proc Natl Acad Sci U S A, 2010. 107(18): p. 8129-34.

11. Watanabe, K., et al., A ROCK inhibitor permits survival of dissociated human embryonic stem cells. Nat Biotechnol, 2007. 25(6): p. 681-6.

12. Deng, J., et al., Targeted bisulfite sequencing reveals changes in DNA methylation associated with nuclear reprogramming. Nat Biotechnol, 2009. 27(4): p. 353-60.

13. Chin, M.H., et al., Induced pluripotent stem cells and embryonic stem cells are distinguished by gene expression signatures. Cell Stem Cell, 2009. 5(1): p. 111-23.

14. Meissner, A., M. Wernig, and R. Jaenisch, Direct reprogramming of genetically unmodified fibroblasts into pluripotent stem cells. Nat Biotechnol, 2007. 25(10): p. 117781.

15. Hu, B.Y., et al., Neural differentiation of human induced pluripotent stem cells follows developmental principles but with variable potency. Proc Natl Acad Sci U S A, 2010. 107(9): p. 4335-40.

16. Nguyen, D.C., et al., Microscale generation of cardiospheres promotes robust enrichment of cardiomyocytes derived from human pluripotent stem cells. Stem Cell Reports, 2014. 3(2): p. 260-8.

17. Amoroso, M.W., et al., Accelerated high-yield generation of limb-innervating motor neurons from human stem cells. J Neurosci, 2013. 33(2): p. 574-86.

18. Takebe, T., et al., Vascularized and functional human liver from an iPSC-derived organ bud transplant. Nature, 2013. 499(7459): p. 481-4.

19. Takebe, T., et al., Generation of a vascularized and functional human liver from an iPSC-derived organ bud transplant. Nat Protoc, 2014. 9(2): p. 396-409.

20. Ungrin, M.D., et al., Reproducible, ultra high-throughput formation of multicellular organization from single cell suspension-derived human embryonic stem cell aggregates. PLoS One, 2008. 3(2): p. e1565.

21. Kinney, M.A., R. Saeed, and T.C. McDevitt, Systematic analysis of embryonic stem cell differentiation in hydrodynamic environments with controlled embryoid body size. Integr Biol (Camb), 2012. 4(6): p. 641-50.

22. Lian, X., et al., Directed cardiomyocyte differentiation from human pluripotent stem cells by modulating Wnt/beta-catenin signaling under fully defined conditions. Nat Protoc, 2013. 8(1): p. 162-75.

23. Wu, X., et al., Productive hepatitis $C$ virus infection of stem cell-derived hepatocytes reveals a critical transition to viral permissiveness during differentiation. PLoS Pathog, 2012. 8(4): p. e1002617.

24. Grandela, C. and E. Wolvetang, hESC adaptation, selection and stability. Stem Cell Rev, 2007. 3(3): p. 183-91.

25. Kurosawa, H., Methods for inducing embryoid body formation: in vitro differentiation system of embryonic stem cells. J Biosci Bioeng, 2007. 103(5): p. 389-98.

26. Itskovitz-Eldor, J., et al., Differentiation of human embryonic stem cells into embryoid bodies compromising the three embryonic germ layers. Mol Med, 2000. 6(2): p. 88-95. 
27. Abbasalizadeh, S., et al., Bioprocess development for mass production of size-controlled human pluripotent stem cell aggregates in stirred suspension bioreactor. Tissue Eng Part C Methods, 2012. 18(11): p. 831-51.

28. Kehoe, D.E., et al., Scalable stirred-suspension bioreactor culture of human pluripotent stem cells. Tissue Eng Part A, 2010. 16(2): p. 405-21.

29. Wang, Y., L. Cheng, and S. Gerecht, Efficient and scalable expansion of human pluripotent stem cells under clinically compliant settings: a view in 2013. Ann Biomed Eng, 2014. 42(7): p. 1357-72.

30. Kempf, H., et al., Controlling expansion and cardiomyogenic differentiation of human pluripotent stem cells in scalable suspension culture. Stem Cell Reports, 2014. 3(6): p. 1132-46.

31. Jing, D., et al., Stem cells for heart cell therapies. Tissue Eng Part B Rev, 2008. 14(4): p. 393-406.

32. Lock, L.T. and E.S. Tzanakakis, Stem/Progenitor cell sources of insulin-producing cells for the treatment of diabetes. Tissue Eng, 2007. 13(7): p. 1399-412.

33. Carpenedo, R.L., C.Y. Sargent, and T.C. McDevitt, Rotary suspension culture enhances the efficiency, yield, and homogeneity of embryoid body differentiation. Stem Cells, 2007. 25(9): p. 2224-34.

34. Sargent, C.Y., et al., Hydrodynamic modulation of embryonic stem cell differentiation by rotary orbital suspension culture. Biotechnol Bioeng, 2010. 105(3): p. 611-26.

35. Shukla, S., et al., Synthesis and organization of hyaluronan and versican by embryonic stem cells undergoing embryoid body differentiation. J Histochem Cytochem, 2010. 58(4): p. 345-58.

36. Gomez, E.W., et al., Tissue geometry patterns epithelial-mesenchymal transition via intercellular mechanotransduction. J Cell Biochem, 2010. 110(1): p. 44-51.

37. Lian, X., et al., Robust cardiomyocyte differentiation from human pluripotent stem cells via temporal modulation of canonical Wnt signaling. Proc Natl Acad Sci U S A, 2012. 109(27): p. E1848-57.

38. Bao, B., et al., Connexon-mediated cell adhesion drives microtissue self-assembly. FASEB J, 2011. 25(1): p. 255-64.

39. Foty, R.A. and M.S. Steinberg, The differential adhesion hypothesis: a direct evaluation. Dev Biol, 2005. 278(1): p. 255-63.

40. Foty, R.A. and M.S. Steinberg, Differential adhesion in model systems. Wiley Interdiscip Rev Dev Biol, 2013. 2(5): p. 631-45.

41. Thavandiran, N., et al., Design and formulation of functional pluripotent stem cellderived cardiac microtissues. Proc Natl Acad Sci U S A, 2013. 110(49): p. E4698-707.

42. Stevens, K.R., et al., InVERT molding for scalable control of tissue microarchitecture. Nat Commun, 2013. 4: p. 1847.

43. Toyofuku, T., et al., Wnt/frizzled-2 signaling induces aggregation and adhesion among cardiac myocytes by increased cadherin-beta-catenin complex. J Cell Biol, 2000. 150(1): p. 225-41.

44. Van Winkle, A.P., I.D. Gates, and M.S. Kallos, Mass transfer limitations in embryoid bodies during human embryonic stem cell differentiation. Cells Tissues Organs, 2012. 196(1): p. 34-47.

45. Purpura, K.A., et al., Systematic engineering of 3D pluripotent stem cell niches to guide blood development. Biomaterials, 2012. 33(5): p. 1271-80.

46. Bauwens, C.L., et al., Control of human embryonic stem cell colony and aggregate size heterogeneity influences differentiation trajectories. Stem Cells, 2008. 26(9): p. 2300-10.

47. Singh, A., et al., Adhesion strength-based, label-free isolation of human pluripotent stem cells. Nat Methods, 2013. 10(5): p. 438-44. 
EsC
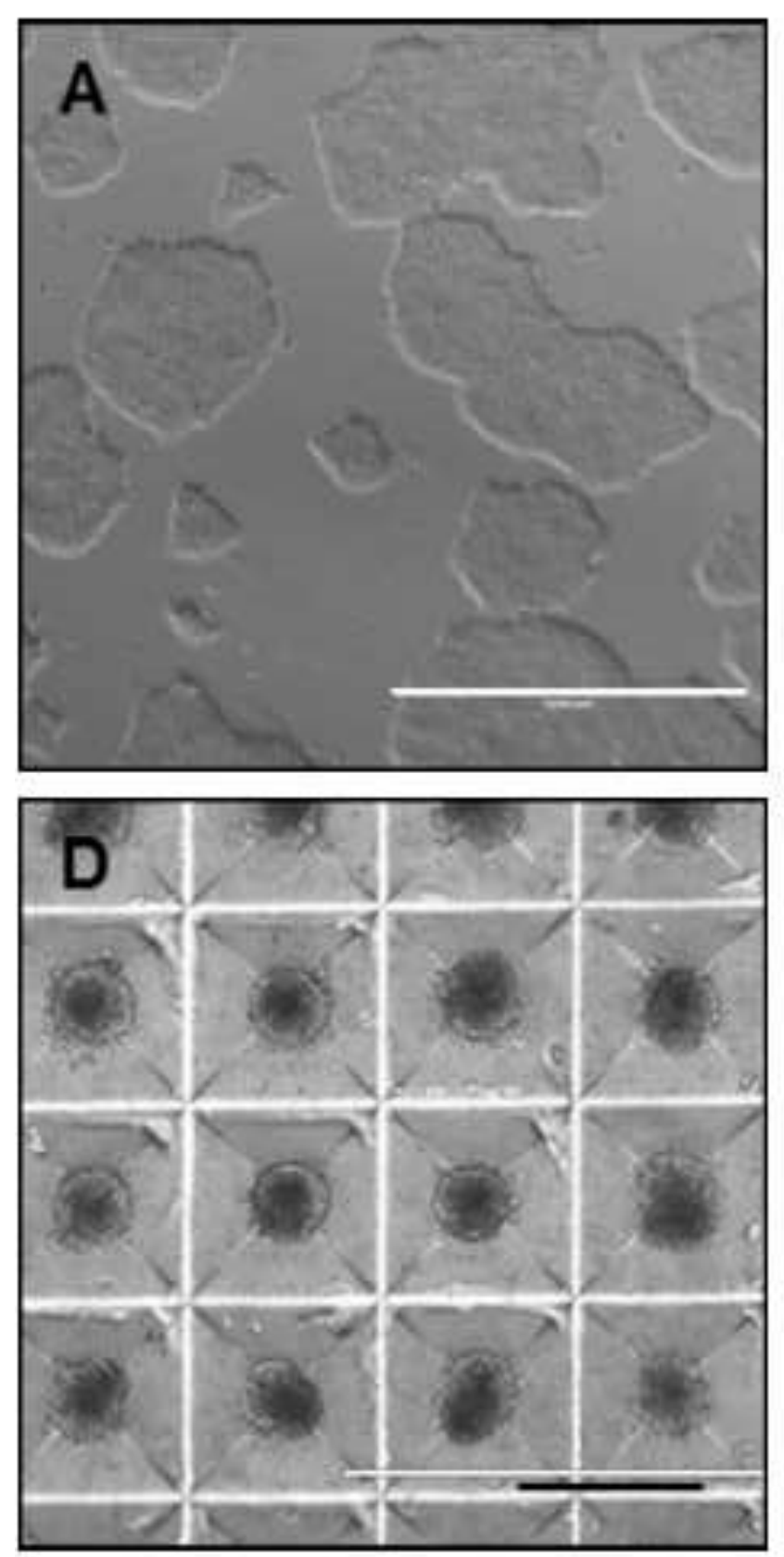

IPSC
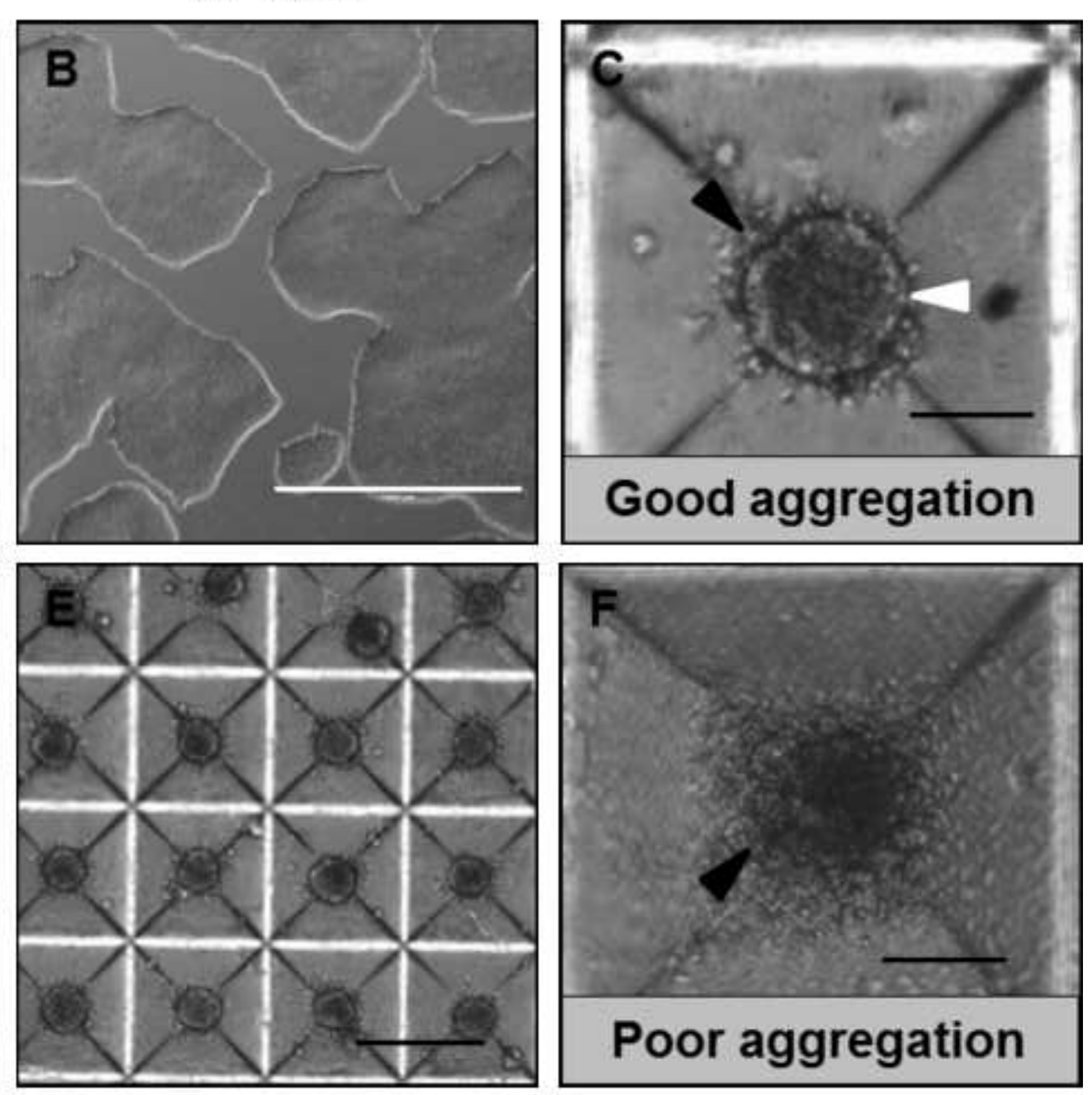

Poor aggregation 

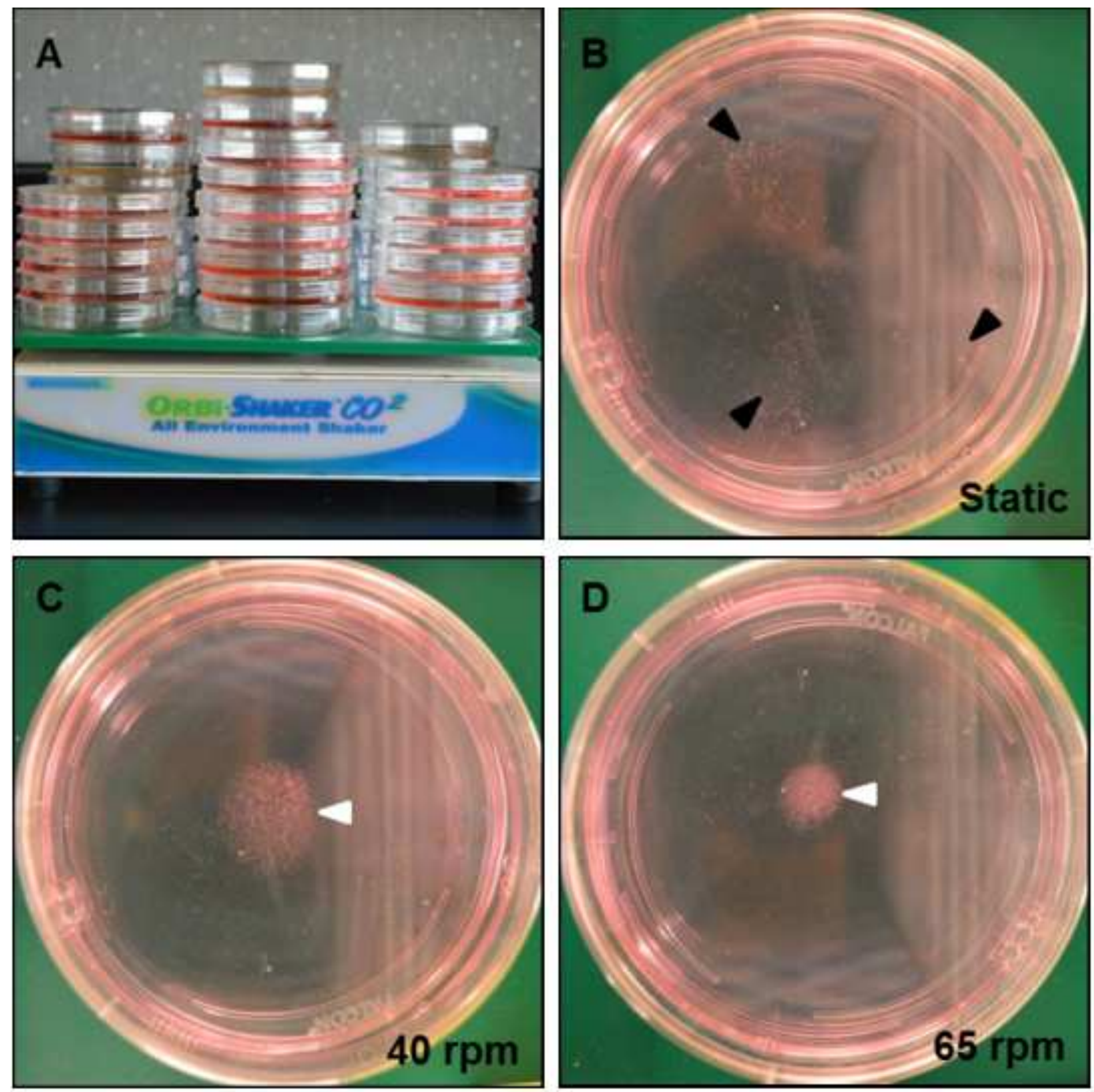

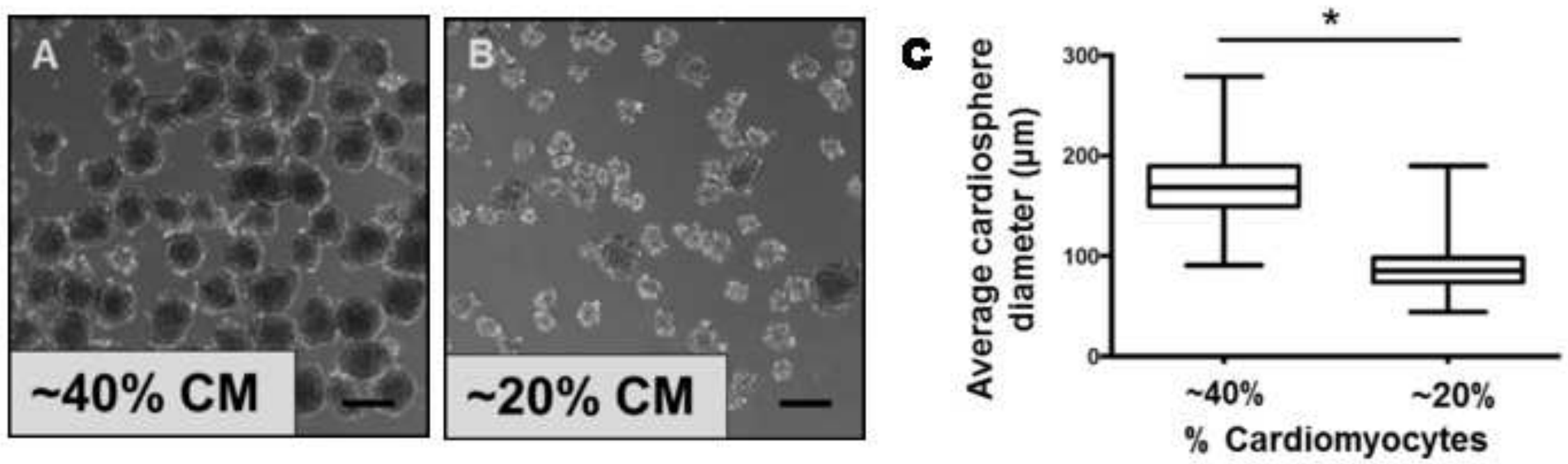

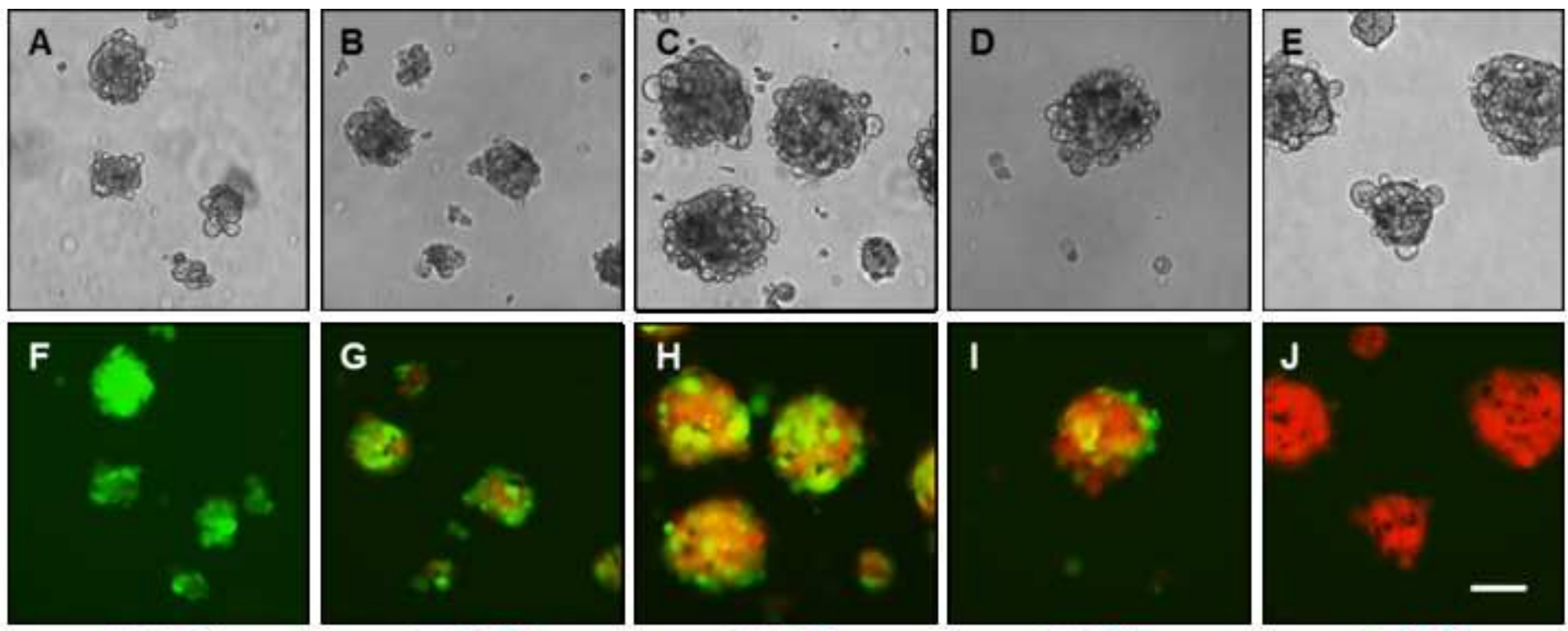

100:0 $75: 25$

$50: 50$

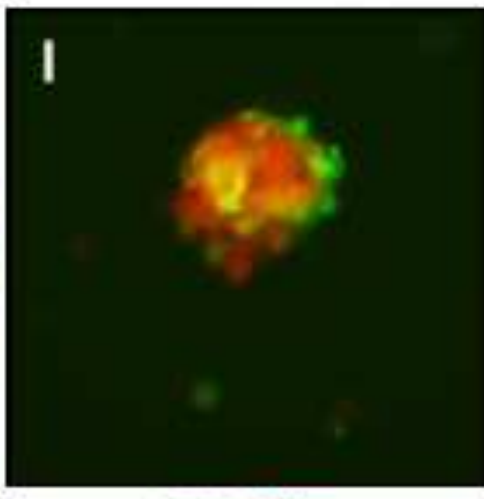

$25: 75$

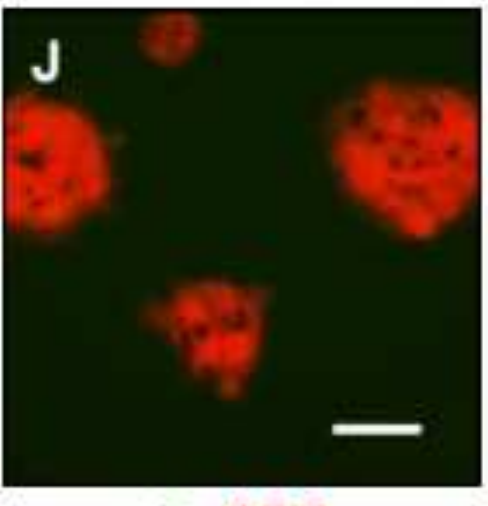

$0: 100$

\section{Cardiomyocytes}


Endoderm

Hepetocytes

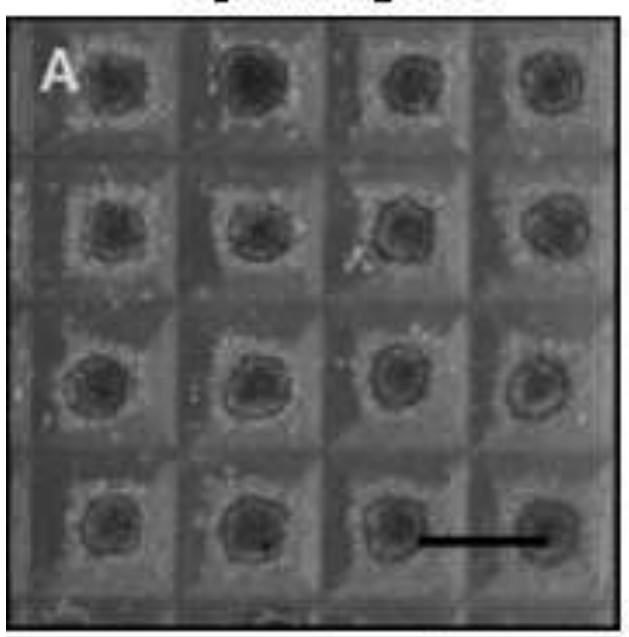

D

D

6.
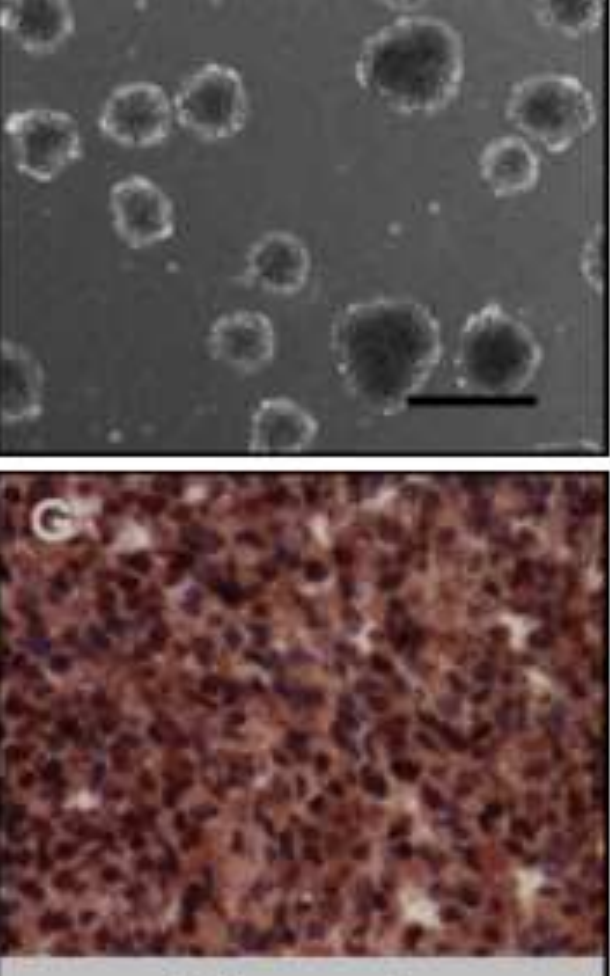

Albumin
Mesioderm

Cardiomyocytes
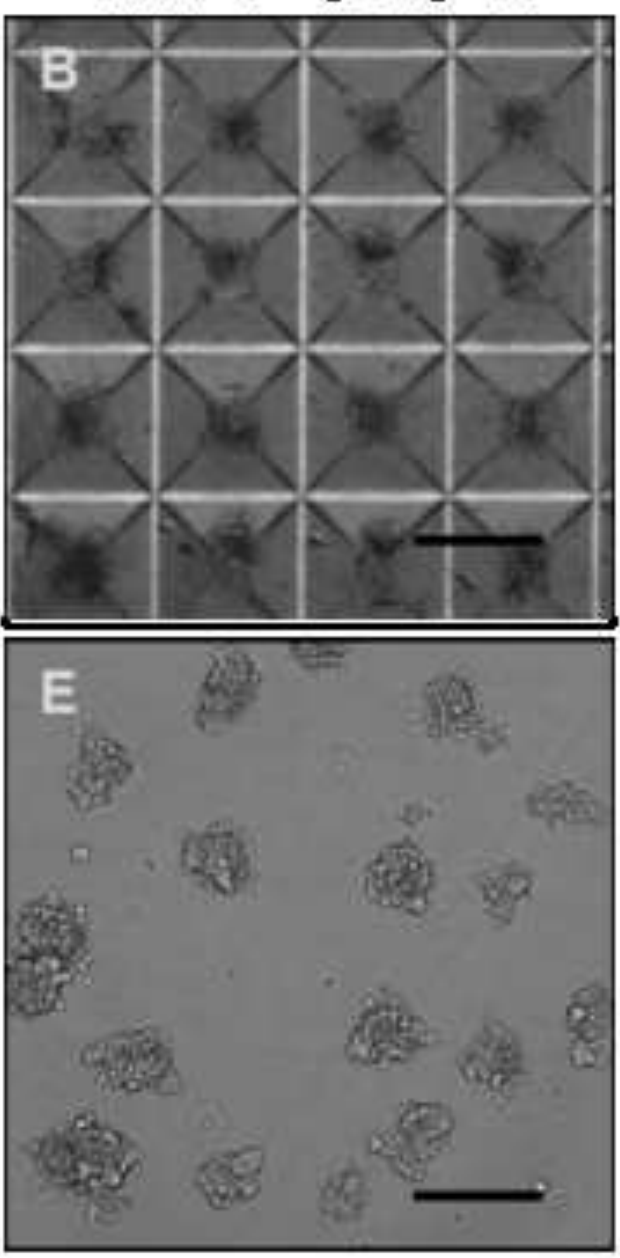

H
Extodem

Motor Neurons
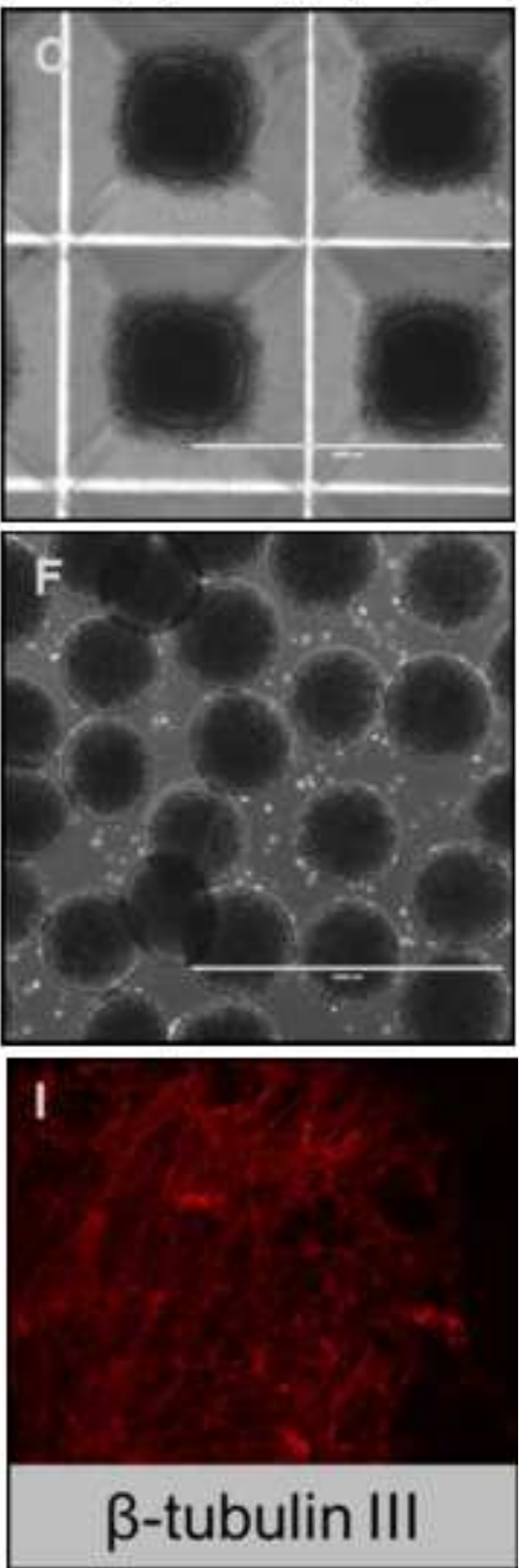